\title{
Global optimization for AVO inversion: a genetic algorithm using a table-based ray-theory algorithm
}

\author{
W. C. Ferreira (UNICAMP \& INCT-GP), F. Hilterman (Geokinectics Inc.), L. A. Diogo (IAG/USP), H. B. Santos (UNICAMP \& \\ INCT-GP), J. Schleicher (UNICAMP \& INCT-GP) and A. Novais (UNICAMP \& INCT-GP)
}

Copyright 2016, SBGf - Sociedade Brasileira de Geofísica.

Este texto foi preparado para a apresentação no Simpósio Brasileiro de Geofísica, Ouro Preto, 25 a 27 de outubro de 2016. Seu conteúdo foi revisado pelo Comitê Técnico do VII SimBGf, mas não necessariamente representa a opinião da SBGf ou de seus associados. É proibida a reprodução total ou parcial deste material para propósitos comerciais sem prévia autorização da SBGf.

\section{Abstract}

Amplitude Variation with Offset (AVO) inversion provides estimates of the P-wave velocity, S-wave velocity and density of a stratified medium. Global optimization is desirable for the inversion to account for the multi-parametric behavior of the AVO inversion which is strongly affected by the initial estimates of the model rock properties. We carried out an analysis to verify the dependency between P-wave, Swave velocity and density in the recovered parameters using empirical relations as constraints. In inversion schemes, the forward modeling is often the most timeconsuming process. To reduce computation time, we have implemented a genetic algorithm using a table-based ray-theory algorithm to allow for a large amount of vertically inhomogeneous models in the global search. Our results show that the genetic algorithm was capable of recovering the physical parameters with good agreement for examples using the empirical constraints. However, it sometimes converged to solutions which were far from the correct answer, but were good models to explain the observed dataset. The forward modeling algorithm has shown excellent performance to be used in global optimization schemes, because it allows the use of a large number of members in the population of the genetic algorithm.

\section{Introduction}

The methodology of AVO has been widely used in the industry as a direct hydrocarbon indicator. A more quantitative interpretation can be achieved by means of AVO inversion for the rock properties. For the forward modeling part, one classically calculates the reflection coefficients for plane waves as a function of incident angle (offset) using the formulas of Knott (1899) and Zoeppritz (1919). For the reverse model, Rosa (1976) derived and verified the ill-posed nature of the inversion of Zoeppritz equations for rock properties, meaning that we have multiple combinations of input to produce the same output. Besides, due to its multi-parametric formulation, the solution space is very complex with many local minima, which makes it difficult to find correct solutions.

For those reasons, Stoffa and Sen (1991), Mallick 1995 and others suggest global optimization schemes to treat the AVO inversion. However, the process of global optimizations requires many forward models which considerably increase the computation time. Many of the works published in the literature use forward modeling algorithms based on the wave equation, which demand great computational power.

This study is based on the premise that unconsolidated sediments with small rock-property changes between layers can be modeled with ray theory without a loss of resolution. For computational ease, we have developed a forward modeling algorithm called table-based ray theory in order to speed up the computation of synthetic seismograms for global optimization. The genetic algorithm is based on findings of Stoffa and Sen (1991) and Sen and Stoffa (1992) and uses the Gardner et al. (1974) and Castagna et al. (1985) empirical relationships as constraints to verify the dependency among the parameters.

Synthetic common-midpoint seismogram: forward modeling

The forward modeling algorithm implemented is a socalled table-based ray tracing. It assumes an Earth model composed of $n$ horizontal isotropic layers. The parametric equations to compute the two-way traveltimes and offset as a function of a constant ray parameter are given by (Slotnick, 1959)

$$
\begin{aligned}
x & =2 \sum_{i=1}^{n} \frac{h_{i} p v_{i}}{\left(1-p^{2} v_{i}^{2}\right)^{1 / 2}} \\
t & =2 \sum_{i=1}^{n} \frac{h_{i}}{v_{i}\left(1-p^{2} v_{i}^{2}\right)^{1 / 2}}
\end{aligned}
$$

where $h_{i}, v_{i}$ and $p$ are, respectively, the layer thickness and velocity of the $i$ th layer, and the ray parameter defined by

$$
p=\frac{\sin \left(\theta_{i}\right)}{v_{i}}
$$

Here $\theta_{i}$ is the angle between the seismic ray and the vertical in the ith layer. Using a sonic log or other vertical velocity information, we build three different two-dimensional tables (offset, traveltime and reflection coefficient) starting with equations 1 and 2

To start the process, the P-wave velocity as a function of depth is transformed to time. The same applies to the Swave velocity and density. For the $2 \mathrm{D}$ traveltime table, there are 90 columns associated with the ray parameter when the incident angle in the first medium varies from $0^{\circ}$ to $89^{\circ}$. The rows are associated with the $t_{o}$ times in increments of the time sample interval. Now, the 2D tables for offset and 
traveltime can easily be determined using equations 1 and 2 respectively. Since the velocity function is sampled in equal increments of the sample rate the same way the $2 \mathrm{D}$ tables are, the computation of the incident angle for each cell in the 2D table is possible with equation 3

With the incidence angle known, the next step is to compute the 2D table of reflection coefficients $R_{P}$ at each interface. This was accomplished with Shuey's approximation (Shuey, 1985) given by

$$
R_{P}(\phi)=A+B \sin ^{2}(\theta)+C \sin ^{2}(\theta) \tan ^{2}(\theta),
$$

where $\phi$ is the incidence angle, $\theta$ is the average of the incidence and transmission angles, and the terms $A, B$ and $C$ are defined as

$$
\begin{aligned}
& A=\frac{1}{2}\left(\frac{\triangle V_{P}}{V_{P}}+\frac{\triangle \rho}{\rho}\right), \\
& B=\frac{1}{2} \frac{\triangle V_{P}}{V_{P}}-2\left(\frac{V_{S}}{V_{P}}\right)^{2}\left(\frac{2 \triangle V_{S}}{V_{S}}+\frac{\triangle \rho}{\rho}\right), \\
& C=\frac{1}{2}\left(\frac{\triangle V_{P}}{V_{P}}\right) .
\end{aligned}
$$

In these expressions, $\triangle V_{P}=V_{P 2}-V_{P 1}, \triangle V_{S}=V_{S 2}-V_{S 1}$, $\triangle \rho=\rho_{2}-\rho_{1}$ are the differences between the $\mathrm{P}$ and $\mathrm{S}$ wave velocity and density values, respectively, across an interface and $V_{P}, V_{S}$ and $\rho$ are their arithmetic averages.

In order to build a synthetic common-midpoint (CMP) section, we define which are the offsets to model and start a search row-by-row inside the offset table looking for the requested offsets. Once the cell is found for the specified offset, we go into the same cell position inside the traveltime and reflectivity tables to place the reflection coefficient from that position at the correct time arrival inside the trace.

Because the algorithm is very simple, it provides the potential of being adapted in the future to study more complex effects such as the stretch in normal moveout (NMO) correction, NMO without stretch (ray-trace NMO correction), array effects which affect the amplitude of the wave received by the streamer, extraction of AVO attributes, polar anisotropic media, etc.

\section{Global optimization approach: Genetic Algorithm}

The genetic algorithm (Figure 1 is a technique used to perform global optimizations based on the natural selection process (Holland, 1975). In order to simulate this process, the algorithm constantly modifies the initial pseudo-random population in order to reach local minima positions. Initially, each member of the pseudo-random population is a potential solution to the problem and after some iterations the genetic algorithm guides the population to the best fit positions.

The algorithm needs to select a percentage of the population to start the reproduction scheme where the crossover and the mutation are the two classic techniques to exchange genetic information between the members and also randomly change the genetic information to provide exploration of the solution space. In order to select the members of the population we need to measure the fitness between each potential solution and the data we want to optimize. For this purpose, we used the objective function

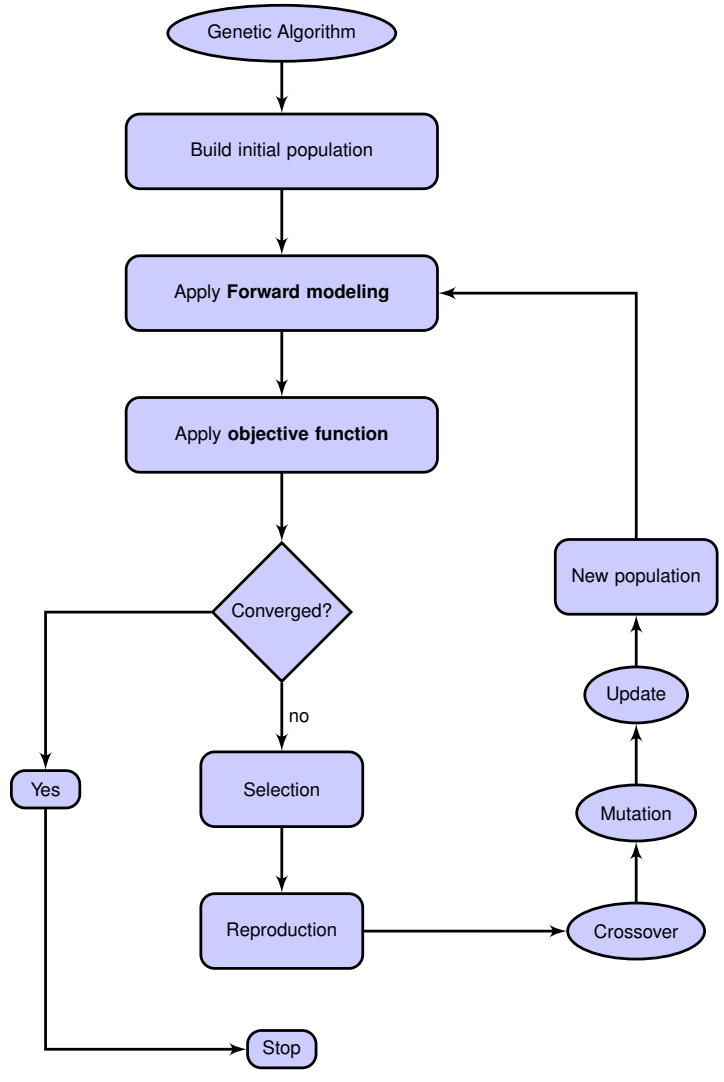

Figure 1: Flowchart of the genetic algorithm.

of Porsani et al. 2000 given by

$$
h=\frac{2 y^{T} x}{y^{T} y+x^{T} x},
$$

where, $x$ and $y$ are the observed and modeled data in the time domain and $x^{T}, y^{T}$ are their transposes. In order to constrain the inverse problem due to the dependency between S-wave velocity and density with $\mathrm{P}$-wave velocity, we used the Gardner (Gardner et al., 1974) and Castagna Castagna et al. 1985 relations to write S-wave velocity and density as a function of $\mathrm{P}$-wave velocity.

\section{Results}

We used a synthetic model in order to verify the global optimization method for AVO inversion and its relationships with the prior information, i.e., the Gardner et al. (1974) and Castagna et al. (1985) constraints, and also to analyze the performance of the investigated forward modeling algorithm. The physical parameters for a simple synthetic model are described in the Table 1 The thickness between the two inner layers was chosen to be small enough to allow interaction between the wavelets.

We modeled a synthetic data set with the table-based raytheory algorithm using the parameters from Table 1 a zero-phase Ricker wavelet with central frequency of $40 \mathrm{~Hz}$, and a time sample rate of $2 \mathrm{~ms}$. These parameters were also used in the modeling for the inversion. In order to perform the global optimization, each CMP gather modeled during each genetic algorithm run is NMO corrected. The parameters used in the genetic algorithm are given by 

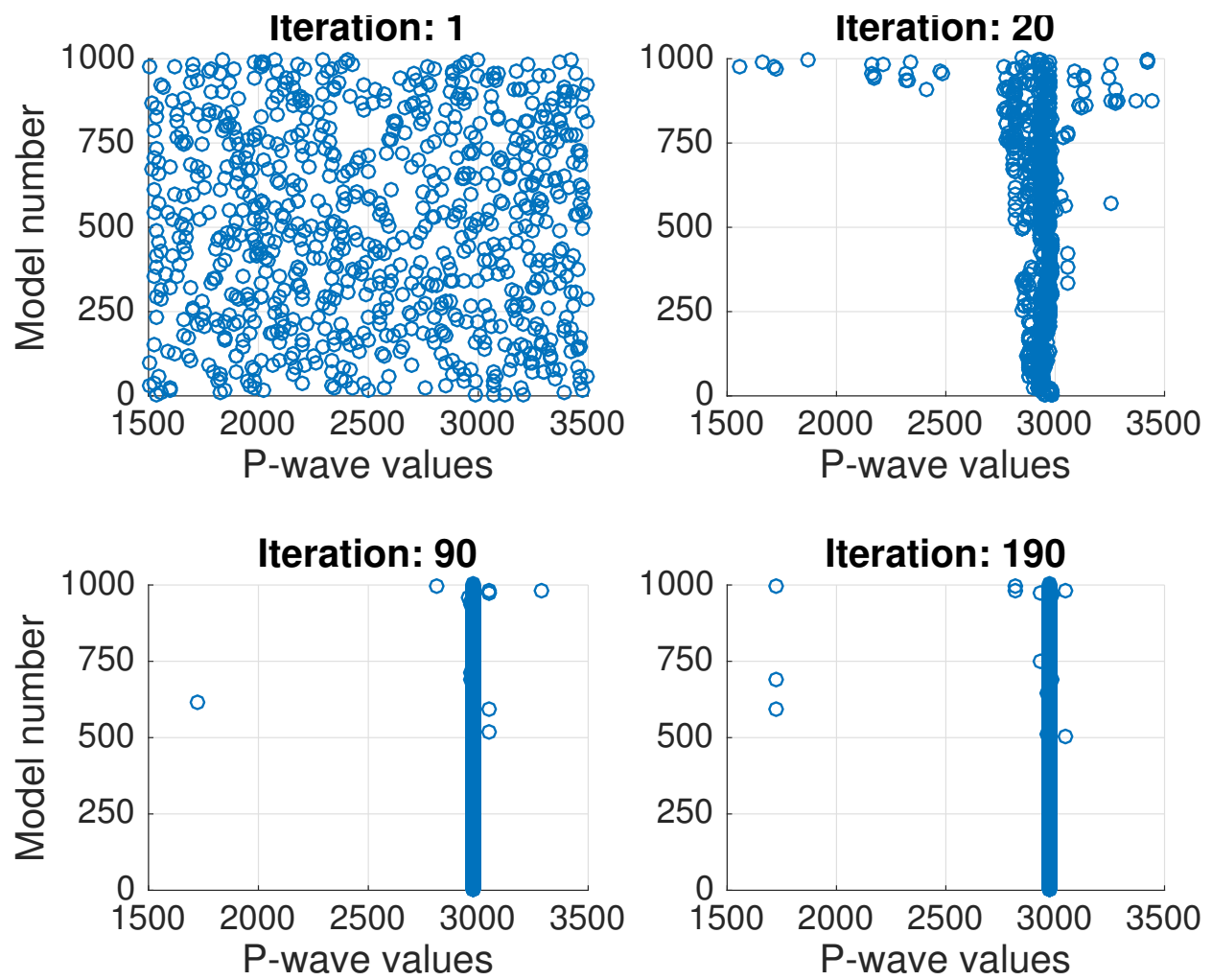

(a)
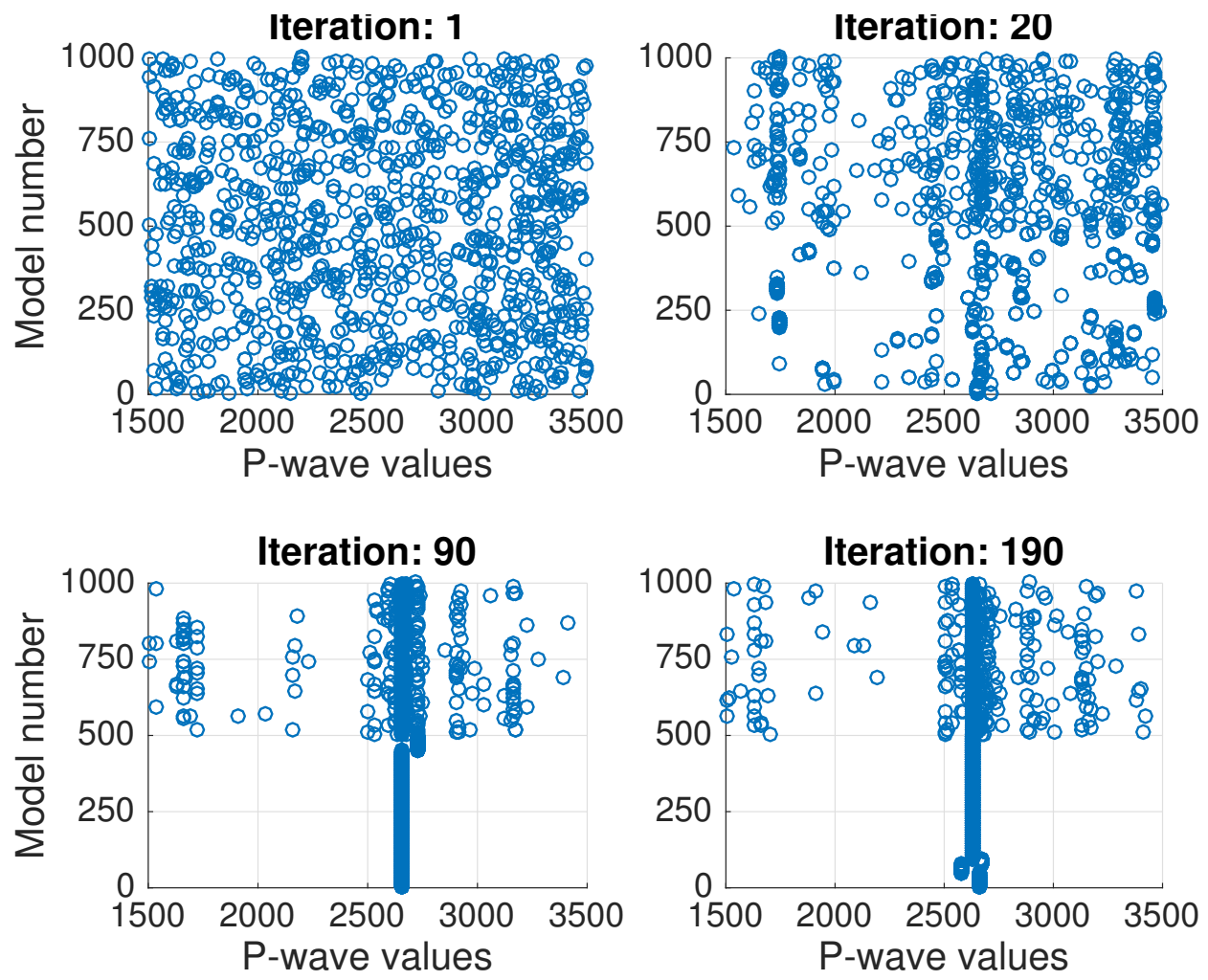

(b)

Figure 2: Evolution of the genetic algorithm solutions for the P-wave velocity parameter in the fourth layer when running with (a) and without (b) the Gardner et al. (1974) and Castagna et al. (1985) constraints. 
Table 1: Model parameters to test the inversion scheme.

\begin{tabular}{ccccc}
\hline Layers & $\begin{array}{c}\text { Thickness } \\
(\mathbf{m})\end{array}$ & $\begin{array}{c}\mathbf{V p} \\
(\mathbf{m} / \mathbf{s})\end{array}$ & $\begin{array}{c}\text { Vs } \\
(\mathbf{m} / \mathbf{s})\end{array}$ & $\begin{array}{c}\text { Density } \\
\left(\mathbf{g} / \mathbf{c}^{\mathbf{3}}\right)\end{array}$ \\
\hline 1 & 1000 & 2000 & 551.72 & 2.07 \\
2 & 50 & 2800 & 1241.38 & 2.25 \\
3 & 50 & 2300 & 810.34 & 2.14 \\
4 & 500 & 3000 & 1413.80 & 2.29 \\
\hline
\end{tabular}

Table 2: Recovered parameters of the model with and without constraints.

\begin{tabular}{|c|c|c|c|c|c|}
\hline \multirow[t]{2}{*}{ Parameters } & \multirow[t]{2}{*}{ Synthetic Model } & \multicolumn{2}{|c|}{$\begin{array}{l}\text { Recovered Model } \\
\text { (with constraints) }\end{array}$} & \multicolumn{2}{|c|}{$\begin{array}{l}\text { Recovered Model } \\
\text { (without constraints) }\end{array}$} \\
\hline & & Parameters & Relative Error (\%) & Parameters & Relative Error (\%) \\
\hline $\begin{array}{l}\text { P-wave } \\
(\mathrm{m} / \mathrm{s})\end{array}$ & $\begin{array}{l}2000 \\
2800 \\
2300 \\
3000\end{array}$ & $\begin{array}{l}2000 \\
2788 \\
2280 \\
2971\end{array}$ & $\begin{array}{l}0.00 \\
0.43 \\
0.87 \\
0.97\end{array}$ & $\begin{array}{l}2002 \\
2699 \\
2394 \\
2658\end{array}$ & $\begin{array}{l}-0.10 \\
3.61 \\
-4.09 \\
11.40\end{array}$ \\
\hline $\begin{array}{l}\text { S-wave } \\
(\mathrm{m} / \mathrm{s})\end{array}$ & $\begin{array}{c}551.72 \\
1241.38 \\
810.34 \\
1413\end{array}$ & $\begin{array}{c}552.71 \\
1231 \\
793 \\
1389\end{array}$ & $\begin{array}{l}-0.07 \\
0.84 \\
2.14 \\
1.75\end{array}$ & \begin{tabular}{c|}
778 \\
1327 \\
1106 \\
1358
\end{tabular} & $\begin{array}{c}-41.01 \\
-6.90 \\
-36.49 \\
3.95\end{array}$ \\
\hline $\begin{array}{l}\text { Density } \\
\left(\mathrm{g} / \mathrm{c}^{3}\right)\end{array}$ & $\begin{array}{l}2.07 \\
2.25 \\
2.14 \\
2.29\end{array}$ & $\begin{array}{l}2.069 \\
2.247 \\
2.134 \\
2.284\end{array}$ & $\begin{array}{l}0.01 \\
0.13 \\
0.24 \\
0.25\end{array}$ & $\begin{array}{l}1.535 \\
1.739 \\
1.541 \\
1.934\end{array}$ & $\begin{array}{l}25.83 \\
22.71 \\
28.01 \\
15.31\end{array}$ \\
\hline Correlation Coef. & 1 & \multicolumn{2}{|c|}{0.999887645} & \multicolumn{2}{|c|}{0.999847412} \\
\hline
\end{tabular}

a selection rate of $50 \%, P_{\text {mutation }}=0.1, P_{\text {update }}=0.47$, $P_{\text {crossover }}=0.90,1000$ members in the population and a stopping criterion of 200 iterations.

To study the quality of the inversion, we concentrate on the parameters of the fourth layer of the model in Table 1 Figure 2 shows the evolution of the genetic algorithm solutions for the inverted $\mathrm{P}$-wave velocity parameter in the fourth layer when running with and without the Gardner et al. (1974) and Castagna et al. (1985) constraints.

As we can see in Figure 2(a), when using the constraints, the algorithm is guiding, already after a few iterations, all the members of the population close to the correct position in the solution space. However, Figure 2(b) shows that without the additional constraints to restrict the solutions, the algorithm is leading a significant part of the population to particular solutions that are not very close to the correct one. Nonetheless, these solutions are good answers to the objective function of the inverse problem as we can see in Table 2

In Table 2 we have compiled the recovered parameters for the models with best value of the objective function that where recovered with and without the constraints on the parameters. In the process, the algorithm generated 301.000 forward synthetics in 25 minutes. Even with more than $20 \%$ of relative error in the average, the best model recovered without the constraints still produces a very similar CMP gather as we can verify by the correlation coefficient value. This is a consequence of the ill-posed nature of the underlying inverse problem as verified by Rosa 1976.

\section{Conclusions}

We have presented a table-based ray-tracing forward modeling algorithm that is very fast in calculating the traveltime and reflection coefficient vs. offset information for many vertically inhomogeneous models. By providing good performance with restricted computer power, this algorithm has shown excellent potential to be used in a genetic global optimization scheme for AVO inversion. In the inversion procedure, parameter constraints were helpful to reach the correct global minimum. However, the result without constraints indicate that the procedure may still converge to incorrect results that satisfy the objective function with quite a low residual. In future research, the genetic algorithm will be formulated inside the Bayesian framework to provide solid statistical information about the distribution of the solutions during the iterations. It is expected to be able to visualize distinguishable concentrations of particular solutions which could be useful to determine possible scenarios for the recovered parameters.

\section{Acknowledgments}

This research was supported by the Brazilian research agencies CAPES, CNPq, FAPESP and FINEP. The first author W. C. Ferreira thanks to CAPES for the Science Without Borders fellowship, Geokinetics and the Allied Geophysical Laboratories. H. B. Santos is grateful to CGG-Brazil, Petrobras, ANP and PRH-PB230 for his fellowships. Additional support for the authors was provided by the sponsors of the Wave Inversion Technology (WIT) Consortium. 


\section{References}

Castagna, J. P., M. L. Batzle, and R. L. Eastwood, 1985, Relationships between compressional-wave and shearwave velocities in clastic silicate rocks: Geophysics, 50, 571-581, doi: 10.1190/1.1441933.

Gardner, G. H. F., L. W. Gardner, and A. R. Gregory, 1974, Formation velocity and density - the diagnostic basics for stratigraphic traps: Geophysics, 39, 770-780, doi: 10.1190/1.1440465.

Holland, J., 1975, Adaptation in natural and artificial systems: an introductory analysis with applications to biology, control, and artificial intelligence: University of Michigan Press.

Knott, C. G., 1899, Reflection and refraction of elastic waves with seismological applications: Philosophical Magazine, 48, 64-97.

Mallick, S., 1995, Model-based inversion of amplitudevariations-with-offset data using a genetic algorithm: Geophysics, 60, 939-954, doi: 10.1190/1.1443860.

Porsani, M. J., P. L. Stoffa, M. K. Sen, and R. K. Chunduru, 2000, Fitness functions, genetic algorithms and hybrid optimization in seismic waveform inversion: Journal of Seismic Exploration, 9, 143-164.

Rosa, L. R., 1976, Extraction of elastic parameters using seismic reflection amplitude with offset variations: Master's thesis, University of Houston.

Sen, M. K., and P. L. Stoffa, 1992, Rapid sampling of model space using genetic algorithms: examples from seismic waveform inversion: Geophysical Journal International, 108, 281-292.

Shuey, R. T., 1985, A simplification of the Zoeppritz equations: Geophysics, 50, 609-614, doi: 10.1190/1.1441936.

Slotnick, M. M., 1959, Lessons in Seismic Computing (Society of Exploration Geophysicists), 1: Society of Exploration Geophysicists.

Stoffa, P. L., and M. K. Sen, 1991, Nonlinear multiparameter optimization using genetic algorithms: Inversion of plane-wave seismograms: Geophysics, 56, 1794-1810, doi: 10.1190/1.1442992.

Zoeppritz, K., 1919, Über Reflexion und Durchgang seismischer Wellen durch Unstetigkeitsflächen: Nachrichten von der Königlichen Gesellschaft der Wissenschaften zu Göttingen, Mathematischphysikalische Klasse, VIIb, 66-84. 